\title{
Mechanism for blob generation in the TORPEX toroidal plasma ${ }^{\text {a) }}$
}

\author{
I. Furno, ${ }^{1, b)}$ B. Labit, ${ }^{1}$ A. Fasoli, ${ }^{1}$ F. M. Poli, ${ }^{1}$ P. Ricci, ${ }^{1}$ C. Theiler, ${ }^{1}$ S. Brunner, ${ }^{1}$ A. Diallo, ${ }^{1}$ \\ J. P. Graves, ${ }^{1}$ M. Podestà, ${ }^{2}$ and S. H. Müller ${ }^{3}$ \\ ${ }^{1}$ Centre de Recherche en Physique des Plasmas, Ecole Polytechnique Fédérale de Lausanne, \\ Association EURATOM-Confédération Suisse, CH-1015 Lausanne, Switzerland \\ ${ }^{2}$ Department of Physics and Astronomy, University of California, Irvine, California 92697, USA \\ ${ }^{3}$ Center for Energy Research, University of California, San Diego, La Jolla, California 92093, USA
}

(Received 9 November 2007; accepted 28 January 2008; published online 11 March 2008)

\begin{abstract}
The mechanism for blob generation is detailed in the toroidal magnetized plasma of the TORPEX device [Fasoli et al., Phys. Plasmas 13, 055902 (2006)] using an experimental configuration, which features a plasma region dominated by a coherent wave and a region on the low field side characterized by the propagation of blobs. Predictions from linearized 2D drift-reduced Braginskii equations are compared with experimental data, revealing the interchange nature of the coherent wave. The dynamics of blob formation is investigated using time-resolved measurements of two-dimensional profiles of electron density, temperature, plasma potential and $\mathbf{E} \times \mathbf{B}$ velocity. Blobs form from radially elongated structures associated with the interchange wave. When a blob is generated, the following sequence of events is observed: 1) A decrease of local pressure gradient length provides an increase of the interchange mode drive; 2) in response, the interchange mode increases in amplitude and expands in the radial direction forming a radially elongated structure from the wave crest; 3) the elongated structure is convected by the $\mathbf{E} \times \mathbf{B}$ flow and is eventually sheared off, forming a blob on the low field side. The dependence of the blob amplitude upon the minimum pressure radial scale length before the blob ejection is also investigated. (C) 2008 American Institute of Physics. [DOI: 10.1063/1.2870082]
\end{abstract}

\section{INTRODUCTION}

At the edge of magnetic confinement devices, such as tokamaks ${ }^{1-3}$ stellarators, ${ }^{4}$ reversed field pinches, ${ }^{5}$ and linear devices, ${ }^{6-8}$ a large fraction of the anomalous particle and heat transport is attributed to the presence of blobs. These are intermittent isolated structures where density and temperature are increased with respect to the surrounding plasma. Blobs extend along the field lines and propagate away from the plasma core resulting in heat and particle transport. Blob transport is convective and may extend to the far Scrape-Off Layer (SOL), typically yielding flat density profiles and a significant particle flux near the wall of the device, affecting divertor heat loads and wall recycling. In addition, a correlation has been found between increased intermittent transport in the SOL and the density limit in tokamaks, suggesting that the transport associated with blobs may play a role in this operational limit. ${ }^{9}$ Recently, it has also been shown that blob transport may affect the overall performance of the ITER. $^{10}$

In recent years, there has been an increasing experimental, theoretical, and numerical simulation effort to understand the physics of blobs (see review article in Ref. 11, and references therein). The identification of the physical mechanism underlying the generation of blobs is of importance for validating first-principle models of edge/SOL transport and may in practice lead to tools for reducing turbulent transport at the plasma edge. Experimental data and numerical simulations suggest that blobs arise from nonlinear instabilities at

\footnotetext{
${ }^{\text {a) }}$ Paper CI1 4, Bull. Am. Phys. Soc. 52, 59 (2007).

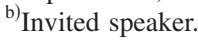

the plasma edge. The generation of blobs is reproduced in a variety of two-dimensional (2D) ${ }^{12,13}$ and three-dimensional ${ }^{14}$ numerical simulations performed with codes commonly used for edge/SOL transport in fusion devices. Recent simulations have shown that blobs can be expelled from interchange turbulence finding agreement with single point measurements from Langmuir probes in TCV plasmas. ${ }^{15}$ Data from gas puff imaging systems ${ }^{16,17}$ have provided indirect evidence that blobs are generated in the vicinity of the last closed flux surface of tokamak plasmas, presumably as a result of interchange instability, and are associated with relaxations of the edge pressure profile. ${ }^{18}$ From the experimental point of view, the investigation of the generation mechanism in fusion devices is hampered by the intrinsic difficulty to have diagnostic access in the region of interest with adequate temporal and spatial resolution. To overcome these limitations, a number of investigations of blob physics have recently been undertaken in basic plasma physics devices, ${ }^{6-8}$ in which full diagnostics access with adequate spatial and temporal resolution can be obtained. Experiments in the linear magnetized helicon device VINETA show a close link between blobs and quasicoherent drift waves. ${ }^{8}$ In the toroidal device TORPEX, ${ }^{19}$ an experimental configuration has been identified, in which blobs with properties similar to those observed in tokamaks are found to originate from the wave crest of a coherent interchange wave. ${ }^{20}$

The present article is an expanded description of the investigation of the blob generation mechanism in the TORPEX device, described in Ref. 21. We study the blob dynamics using time-resolved 2D profiles of electron density and temperature, plasma potential, and $\mathbf{E} \times \mathbf{B}$ velocity, which are 
obtained using a modified conditional sampling (CS) technique. The results show that blobs form from radially elongated structures that are sheared off by the $\mathbf{E} \times \mathbf{B}$ flow. These structures develop from an interchange wave that increases in amplitude and extends radially in response to a decrease of the radial pressure scale length. We discuss the dependence of the blob amplitude upon the pressure radial scale length.

The remainder of the article is organized as follows: In Sec. II, the experimental setup and the time averaged profiles of the basic plasma parameters are presented. In Sec. III, the nature of plasma fluctuations is investigated using both spectral and statistical methods. In Sec. IV, we discuss the blob generation mechanism while conclusions are summarized in Sec. V. The modified CS technique is detailed in the Appendix.

\section{EXPERIMENTAL SETUP AND TIME-AVERAGED PROFILES}

The experiments are performed in the toroidal device TORPEX (major radius $R=1 \mathrm{~m}$, minor radius $a=0.2 \mathrm{~m}$ ) using hydrogen plasmas produced by means of microwaves in the Electron Cyclotron (EC) range of frequencies. ${ }^{22} \mathrm{~A}$ low level of EC power $P_{\mathrm{EC}} \approx 400 \mathrm{~W}$ is used. A vertical magnetic field $B_{z}=2.3 \mathrm{mT}$ is superposed to a toroidal magnetic field of $B_{t}=76 \mathrm{mT}$ on axis thus resulting in helicoidal magnetic field lines with both ends terminating on the torus vessel. This experimental setup gives rise to a plasma source profile localized on the high-field side, which insures negligible plasma production for $r \geqslant 5 \mathrm{~cm}$ and results in a vertically elongated plasma configuration. ${ }^{22}$ Figures $1(a)-1(c)$ show $2 D$ profiles of the time-averaged (indicated by the overbar symbol) electron pressure, $\bar{p}_{e}=\bar{n}_{e} \bar{T}_{e}$, plasma potential, $\bar{V}_{\mathrm{pl}}$, and $\mathbf{E} \times \mathbf{B}$ velocity, $\overline{\mathbf{v}}_{E \times B}$, obtained from Langmuir probe measurements. The plasma potential is computed from $\bar{V}_{\mathrm{pl}}=\bar{V}_{\mathrm{fl}}$ $+\mu \bar{T}_{e} / e$, where $\bar{V}_{\mathrm{fl}}$ and $\bar{T}_{e}$ are floating potential and electron temperature, and the coefficient $\mu=3.1 \pm 0.6$ is determined experimentally. ${ }^{22}$ The $\mathbf{E} \times \mathbf{B}$ velocity is evaluated from $\overline{\mathbf{v}}_{E \times B}=\left(-\nabla \bar{V}_{\mathrm{pl}} \times \mathbf{B}\right) / B^{2}$, with $B=\sqrt{B_{z}^{2}+B_{t}^{2}}$.

The $z$-averaged radial profiles of $\bar{p}_{e}, \bar{V}_{\mathrm{pl}}$, and the $z$ component of the $\mathbf{E} \times \mathbf{B}$ velocity, $\bar{v}_{z, E \times B}$, are shown in Figs. 1(d)-1(f). The profiles of pressure and plasma potential peak in the high field side region and decrease towards the low field side. The $\mathbf{E} \times \mathbf{B}$ velocity field in Fig. 1(c) is almost vertical for $r \gtrsim 0$ with a radially sheared $z$ component $\bar{v}_{z, E \times B}$. The inverse equilibrium radial pressure gradient scale length, defined as $\left|\bar{L}_{p e}^{-1}\right|=\left|\nabla_{r} \bar{p}_{e}\right| / \bar{p}_{e}$, has a maximum on a vertical line at $r \approx 1 \mathrm{~cm}$, Fig. 1(a). Within the experimental uncertainty, this corresponds to the position of the maximum $\left|\bar{v}_{z, E \times B}\right|$, represented by a dashed line in Fig. 1(b). On this line on the midplane, $\bar{n}_{e} \approx 8 \times 10^{15} \mathrm{~m}^{-3}, \bar{T}_{e} \approx 3.7 \mathrm{eV},\left|\bar{L}_{p e}^{-1}\right| \approx 15 \mathrm{~m}^{-1}$, and $\bar{v}_{z, E \times B} \approx 1200 \mathrm{~ms}^{-1}$. The $\mathbf{E} \times \mathbf{B}$ velocity shear is maximum on a vertical line at $r \approx 3 \mathrm{~cm}$, where $\left|\partial \bar{v}_{z, E \times B} / \partial r\right| \approx 3$ $\times 10^{4} \mathrm{~s}^{-1}$ at midplane.
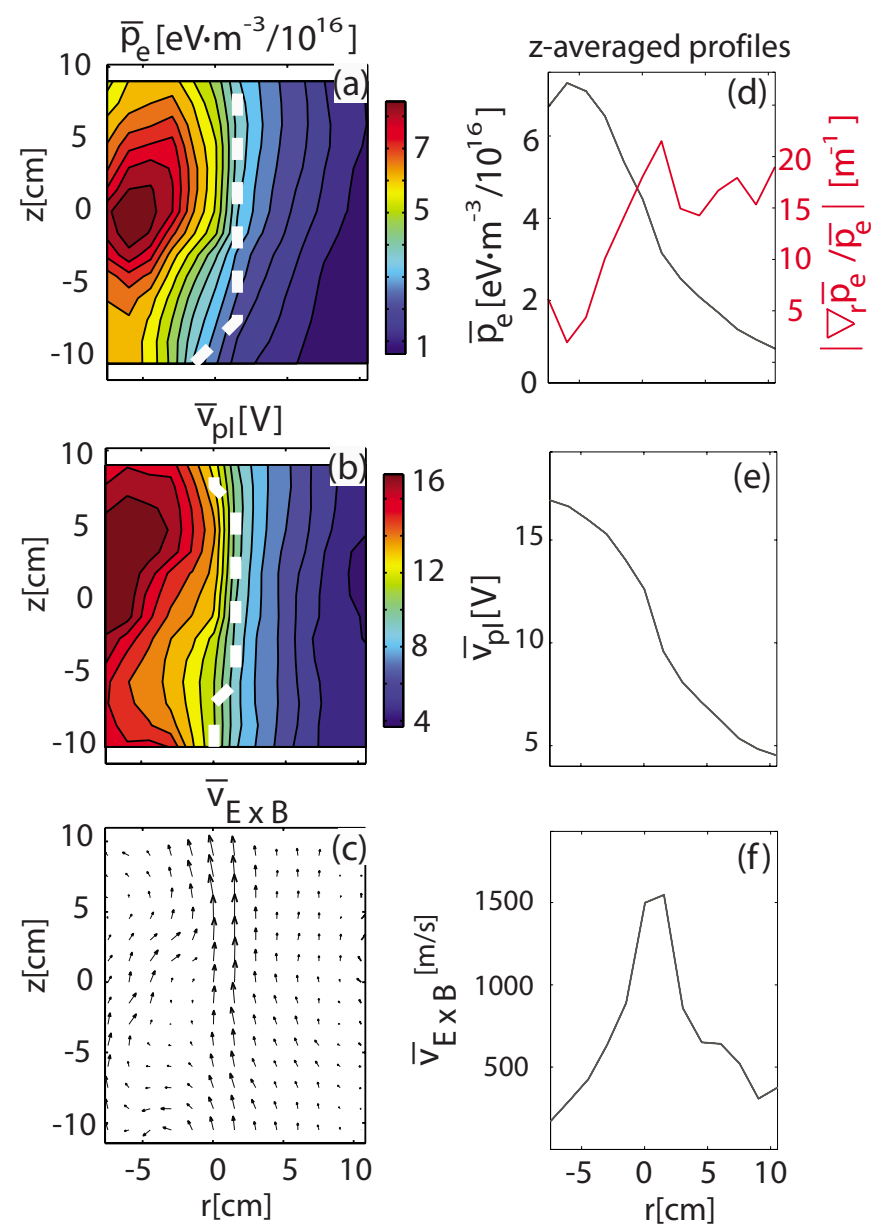

FIG. 1. (Color online) Left panels: 2D poloidal profiles of the time-averaged plasma pressure, plasma potential, and $\mathbf{E} \times \mathbf{B}$ velocity from Langmuir probe measurements. Dashed lines indicate the position of (a) maximum $\left|\bar{L}_{p e}^{-1}\right|$ and (b) maximum of the radial gradient of the plasma potential. Right panels: Time- and $z$-averaged radial profiles.

\section{PLASMA FLUCTUATION PROPERTIES}

The present configuration is characterized by two distinct poloidal regions with different plasma dynamics: 1) A main plasma region for $-5 \leq r \leq 5 \mathrm{~cm}$ which is dominated by a coherent interchange wave; 2) a region on the low-field side for $r \gtrsim 5 \mathrm{~cm}$ with negligible plasma production, dubbed source-free region, characterized by the propagation of plasma blobs, resulting in intermittent transport events. In this section, we discuss spectral and statistical properties of plasma fluctuations in the two regions using ion saturation signals, $I_{\mathrm{sat}}$, from an array of 86 Langmuir probes, dubbed HEXTIP, which provides a complete coverage of the poloidal cross section. A detailed description of HEXTIP can be found in Ref. 23. Figure 2 shows typical $I_{\text {sat }}$ signals measured at the two spatial locations indicated in Fig. 2(h) together with the respective probability distribution function (pdf), and power spectral density. In Figs. 2(g) and 2(h), the 2D profiles of skewness $S$ and kurtosis $K$ (normalized third and fourth order moments of the probability density function) of the $I_{\text {sat }}$ signals show that the character of the fluctuations changes significantly across the radius of the device. 


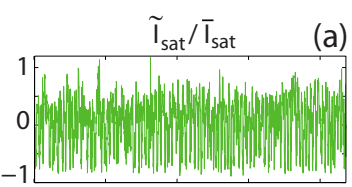

(d)
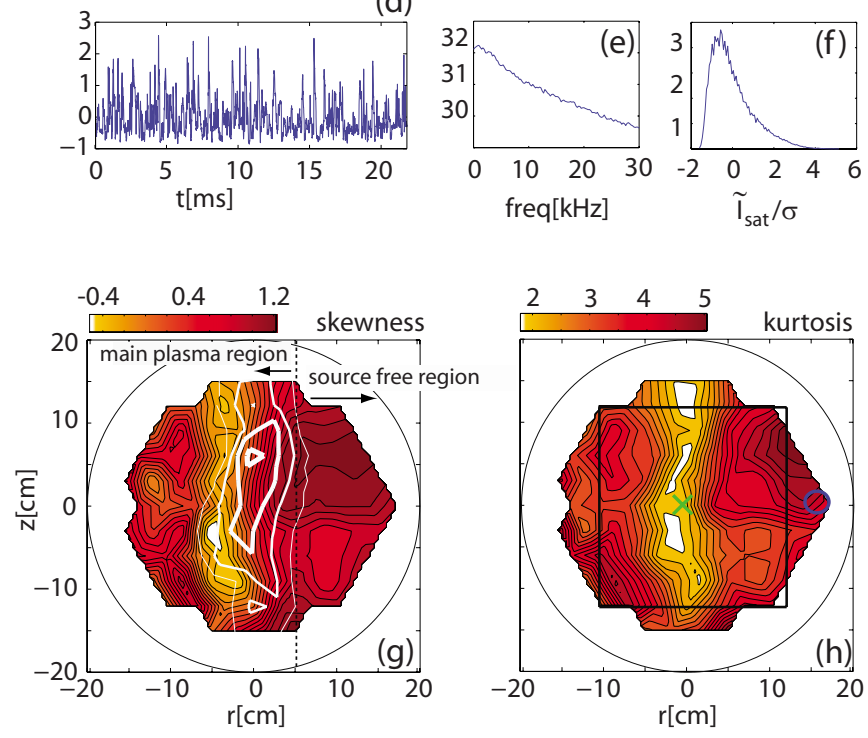

FIG. 2. (Color online) Time series of ion saturation signals (a), (d) at the two locations indicated in Fig. 2(h) show coherent fluctuations in the main plasma region (cross) and intermittent bursts at the edge (circle). Also shown are the respective probability distribution functions in frames (c), (f) and the power spectral densities in frames (b), (e). (g), (h) 2D poloidal profiles of skewness and kurtosis of ion saturation signals. In (g) the profile of the fluctuation power in the frequency range $3.9 \pm 0.5 \mathrm{kHz}$ is shown in white and localizes the interchange wave. Contours correspond to $90 \%$, $60 \%, 30 \%$, and $10 \%$ of the maximum energy. The rectangle in (h) shows the region where the generation mechanism is investigated.

\section{A. Main plasma region: Interchange wave}

In the main plasma region, Fig. 2(a), $\widetilde{I}_{\text {sat }}$ fluctuations are dominated by coherent oscillations with relatively low amplitude $\left|\widetilde{I}_{\text {sat }} / \bar{I}_{\text {sat }}\right| \lesssim 1$ (in the following the over-tilde symbol indicates a fluctuating quantity, i.e., $\left.\widetilde{I}_{\text {sat }}=I_{\text {sat }}-\bar{I}_{\text {sat }}\right)$. This is confirmed by the power spectral density in Fig. 2(b) that peaks at a frequency of $\approx 3.9 \mathrm{kHz}$. The pdf in Fig. 2(c) is double-humped with $S \approx 0$ and $K \approx 2$, as expected for coherent fluctuations. The $2 \mathrm{D}$ profile of the spectral power in the range $f=3.9 \pm 0.5 \mathrm{kHz}$ is shown in Fig. $2(\mathrm{~g})$ and peaks around the position of maximum $\left|\bar{L}_{p e}^{-1}\right|$, where it represents approximately $50 \%$ of the total spectral power of the fluctuating signal. Using a two-point correlation technique ${ }^{24,25}$ we measure a vertical wave number $k_{z} \approx 30.6 \pm 10 \mathrm{~m}^{-1}$ and an upper bound for the parallel wave number $k_{\|}^{\max } \approx 0.046 \mathrm{~m}^{-1}$ at the midplane at $r=1 \mathrm{~cm}$, where the fluctuations in the frequency range have their maximum. The waves propagate upwards with a velocity of $\approx 1200 \mathrm{~ms}^{-1}$, consistent with the time-averaged $\mathbf{E} \times \mathbf{B}$ velocity profile at the midplane.

Using the linearized 2D drift-reduced Braginskii equations (see, e.g., Ref. 26), we show that an interchange mode with $k_{\|}=0$, driven by pressure gradient and curvature and convected by the $\mathbf{E} \times \mathbf{B}$ velocity, is unstable in the region where the mode is experimentally observed. The fluid equations can be written as

$$
\begin{aligned}
\frac{\partial \tilde{n}_{e}}{\partial t}= & \frac{1}{B}\left(\frac{\partial \bar{V}_{\mathrm{pl}}}{\partial r} \frac{\partial \tilde{n}_{e}}{\partial z}-\frac{\partial \widetilde{V}_{\mathrm{pl}}}{\partial z} \frac{\partial \bar{n}_{e}}{\partial r}\right) \\
& +\frac{2}{e R B}\left(\bar{n}_{e} \frac{\partial \tilde{T}_{e}}{\partial z}+\bar{T}_{e} \frac{\partial \tilde{n}_{e}}{\partial z}-e \bar{n}_{e} \frac{\left.\partial \widetilde{V}_{\mathrm{pl}}\right)}{\partial z}\right), \\
\frac{\partial \nabla_{\perp}^{2} \tilde{V}_{\mathrm{pl}}}{\partial t}= & \frac{1}{B}\left(\frac{\partial \bar{V}_{\mathrm{pl}}}{\partial r} \frac{\partial \nabla_{\perp}^{2} \tilde{V}_{\mathrm{pl}}}{\partial z}-\frac{\partial \widetilde{V}_{\mathrm{pl}}}{\partial z} \frac{\left.\partial \nabla_{\perp}^{2} \bar{V}_{\mathrm{pl}}\right)}{\partial r}\right) \\
& +\frac{2 B}{m_{i} R}\left(\frac{\bar{T}_{e}}{\bar{n}_{e}} \frac{\partial \tilde{n}_{e}}{\partial z}+\frac{\partial \tilde{T}_{e}}{\partial z}\right), \\
\frac{\partial \tilde{T}_{e}}{\partial t}= & \frac{1}{B}\left(\frac{\partial \bar{V}_{\mathrm{pl}}}{\partial r} \frac{\partial \tilde{T}_{e}}{\partial z}-\frac{\partial \tilde{V}_{\mathrm{pl}}}{\partial z} \frac{\partial \bar{T}_{e}}{\partial r}\right) \\
& +\frac{4}{3 e R B}\left(\frac{7}{2} \bar{T}_{e} \frac{\partial \tilde{T}_{e}}{\partial z}+\frac{\bar{T}_{e}^{2}}{\bar{n}_{e}} \frac{\partial \tilde{n}_{e}}{\partial z}-e \bar{T}_{e} \frac{\partial \tilde{V}_{\mathrm{pl}}}{\partial z}\right) .
\end{aligned}
$$

Here the over-tilde symbol indicates a small perturbation of a quantity around the equilibrium and $m_{i}$ is the ion mass. Equations (1)-(3) take into account curvature and gradient of the magnetic field, describe modes with $k_{\|}=0$, and neglect $T_{i}$ and electromagnetic effects. We note that, since $\nabla_{\perp}^{2}=\partial^{2} / \partial r^{2}$ $+\partial^{2} / \partial z^{2}$, the system of Eqs. (1)-(3) results in a second order differential equation in $r$. We take $\tilde{n}_{e}=\widetilde{n}_{e}(r) \exp \left(\gamma t+i k_{z} z\right)$, and similarly for $\tilde{V}_{\mathrm{pl}}$ and $\tilde{T}_{e}$. The fluctuations are assumed to vanish at the boundaries of the experimentally measured area. The experimental $z$-averaged profiles of $\bar{n}_{e}(r), \bar{T}_{e}(r)$, and $\bar{V}_{\mathrm{pl}}(r)$ in Figs. 1(d) and 1(e) are used as equilibrium conditions. We compute numerically the eigenmodes, $\tilde{n}_{e}(r)$, $\tilde{T}_{e}(r)$, and $\tilde{V}_{p l}(r)$, and the eigenvalues $\gamma$ for $k_{z}=2 \pi / \Delta$, where $\Delta=2 \pi R B_{z} / B_{t} \approx 18 \mathrm{~cm}$ is the return distance of a field line in the poloidal plane, which constrains the minimum perpendicular wavelength of the interchange mode. The mode with the largest growth rate (i.e., the real part of $\gamma$ ) has $\gamma=7.96$ $\times 10^{4}+i \times 4.03 \times 10^{4} \mathrm{~s}^{-1}$, which corresponds to a real frequency of $\approx 6 \mathrm{kHz}$ in the laboratory frame. This is mostly determined by the $\mathbf{E} \times \mathbf{B}$ frequency $f_{E \times B}=\bar{v}_{E \times B} k_{z} / 2 \pi$, which is much greater than the diamagnetic and curvature frequencies and is very sensitive to the details of the potential profile. In Fig. 3, we show amplitude and phase of the associated eigenmodes. The maximum mode amplitude is localized at $r \approx 1 \mathrm{~cm}$, consistently with experimental data. The phase shift between density and potential at the position of maximum amplitude is $\approx 75^{\circ}$.

\section{B. Source-free region: Blobs}

Across the region of maximum mode amplitude moving radially outwards, the pdfs change from double-humped to positively skewed, Fig. 2(f), and exhibit universal statistical properties with strong similarities with observations in the tokamak SOL. ${ }^{27}$ In the source-free region, $I_{\text {sat }}$ fluctuations have an intermittent character with positive bursts of peak amplitudes $\left|\tilde{I}_{\text {sat }} / \bar{I}_{\text {sat }}\right| \approx 3$. In Fig. 2(e), the fluctuation spectrum is broad and exempt from coherent modes. The skewness profile in Fig. 2(g) remains close to the skewness pro- 

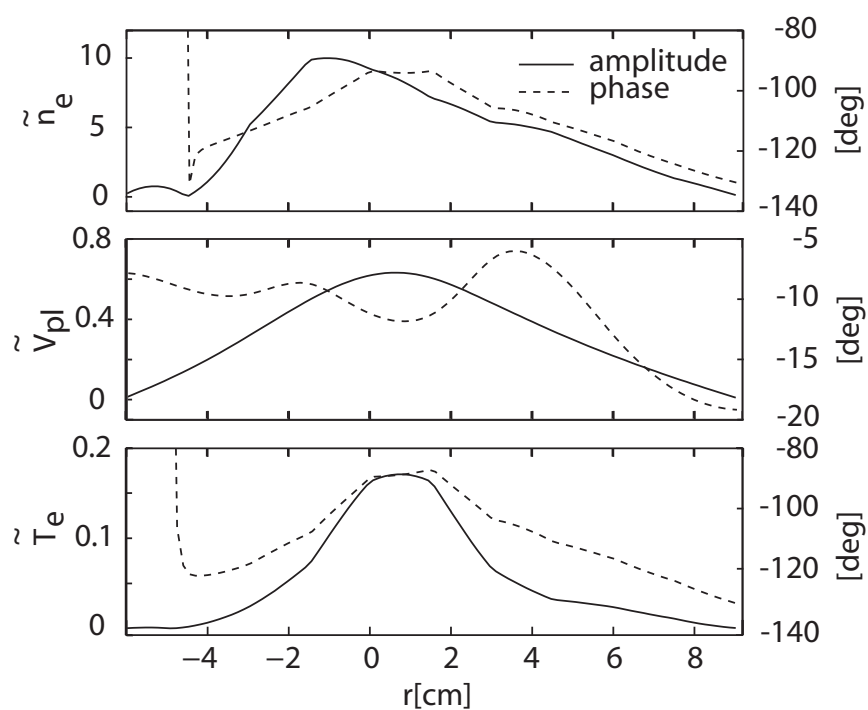

FIG. 3. Radial profiles of the eigenfunction for the fastest growing mode from Eqs. (1)-(3) with $k_{\|}=0$ and $k_{z}=2 \pi / \Delta$, where $\Delta=18 \mathrm{~cm}$, taking into account time- and $z$-averaged profiles of temperature, density and plasma potential of Figs. 1(d)-1(f). Shown are the amplitude (solid line) and phase (dashed line) of the mode .

files measured across the SOL in tokamak plasmas ${ }^{1}$ and suggests the presence of blobs transporting particles and heat into the source-free region.

Due to the intermittent character of the fluctuations in the source-free region, a probabilistic approach is used to investigate their nature, based on a pattern-recognition method combined with statistical analysis. ${ }^{28}$ This method uses $I_{\text {sat }}$ signals from HEXTIP to identify and track positive and negative plasma structures, defined, respectively, as regions where $\widetilde{I}_{\text {sat }}>I_{\text {tresh }}$ and $\widetilde{I}_{\text {sat }}<I_{\text {tresh. }}$. Figure 4 shows the mean velocity field of (a) positive and (b) negative plasma structures identified for $I_{\text {tresh }}=4.5 \mathrm{~mA}$ and lasting between $40 \mu \mathrm{s}$ and $200 \mu \mathrm{s}$. A positive structure example is shown in gray in Fig. 4(a). In the main plasma region, the trajectory patterns for positive and negative structures are very similar consistently with the presence of the wave, while in the source-free region only positive structures - the blobs-have a significant probability of occurrence. As observed in Ref. 20 for slightly different experimental parameters and for a helium plasma, blobs originate from crests of the interchange wave. In Fig. 4(a), the gray structure shears off and generates two structures (black line). The black structure at the low field side is a newly born blob. As shown by the trajectory pattern of the positive structures in Fig. 4(a), blobs propagate outwards in the source-free region as individual coherent structures over distances of the order of the minor radius.

\section{INVESTIGATION OF THE MECHANISM FOR BLOB GENERATION}

Time-resolved 2D profiles of $n_{e}, T_{e}, V_{\mathrm{pl}}$, and velocity fields are required to study the mechanism for blob generation. These are not provided by HEXTIP measurements, but from a conditional sampling method (described in details in the Appendix) using two Langmuir probes. The first probe is

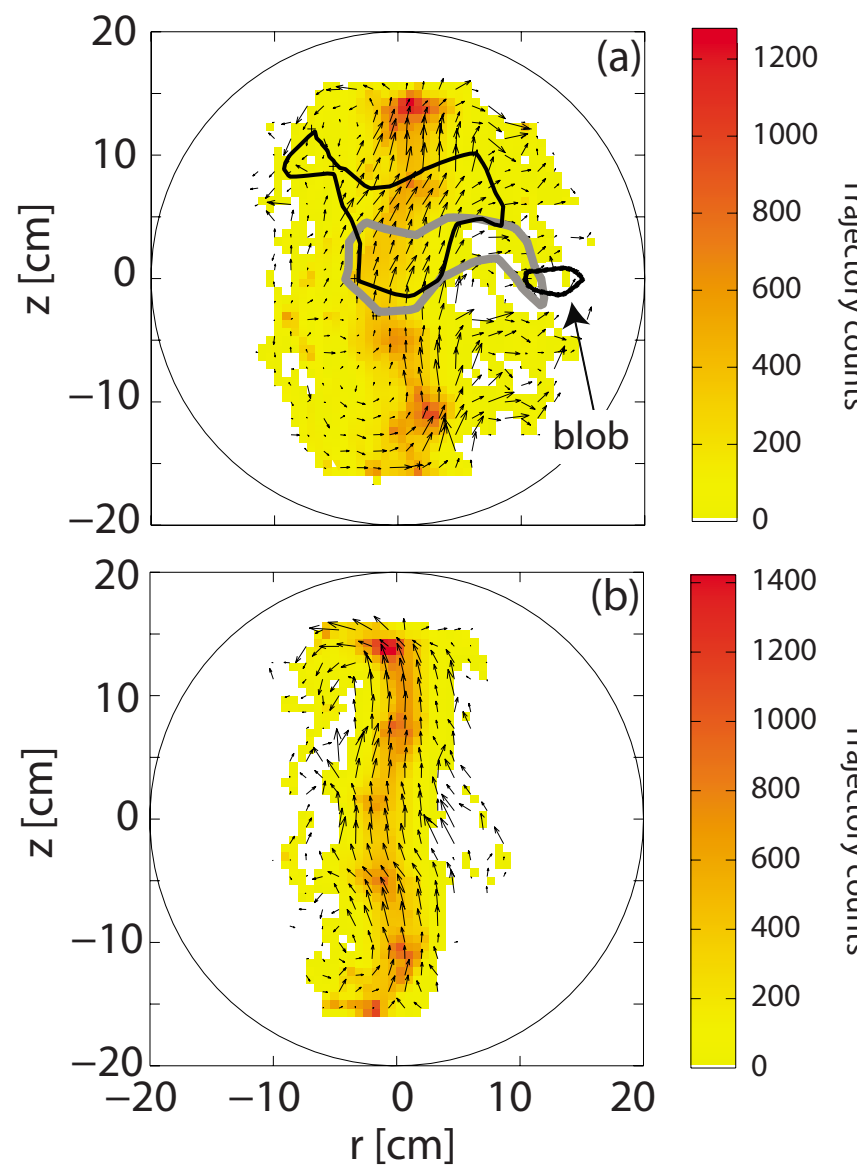

FIG. 4. (Color online) Poloidal distribution of trajectories followed by positive (a) and negative (b) structures, which last between $40 \mu$ s and $200 \mu \mathrm{s}$. The local average velocity of the structures is indicated by arrows. As an example, a single positive structure is shown in gray, which $28 \mu$ s later is sheared off and generates two structures (black line). The black structure on the low field side is a newly born blob.

an eight-tip, L-shaped Langmuir probe array which is moved between discharges over a $25 \times 20 \mathrm{~cm}^{2}$ section in the $r-z$ plane, Fig. 2(h). The second Langmuir probe, dubbed reference probe, is left fixed at the plasma edge $(r=14 \mathrm{~cm}, z=0)$ and toroidally displaced by $90^{\circ}$ from the measuring plane of the Langmuir probe array, Fig. 5(b). The reference probe measures an ion saturation signal $I_{\text {ref }}$, which is used to detect and select blobs of different amplitudes.

Figure 5 illustrates the results of the conditional sampling of $\approx 600$ blobs for the class of blobs defined by $4.5 \sigma$ $\leqslant \widetilde{I}_{\text {ref }} \leqslant 4.7 \sigma$, where $\sigma$ is the standard deviation of the reference signal. Figure 5(a) shows the time evolution of $\widetilde{n}_{e}$ in the mode region (dashed red line) and in the source free region (continuous black line). Figure 5 shows $2 \mathrm{D}$ profiles of $\widetilde{n}_{e}$ in panels (b)-(e) and of $\widetilde{V}_{\text {pl }}$ in panels (f)-(i) at four different times during the ejection of the blob together with the total $\mathbf{E} \times \mathbf{B}$ velocity field. The coherent structures in Figs. 5(b) and 5(c) are identified with the interchange wave, based on their spatio-temporal properties. Comparison between plasma density and plasma potential fluctuations yields a phase shift in the range of $30^{\circ}-45^{\circ}$ with $\widetilde{V}_{\mathrm{pl}}$ leading $\widetilde{n}_{e}$. The difference between this value and the value for the phase shift computed in Sec. III A may be attributed to the effect of 

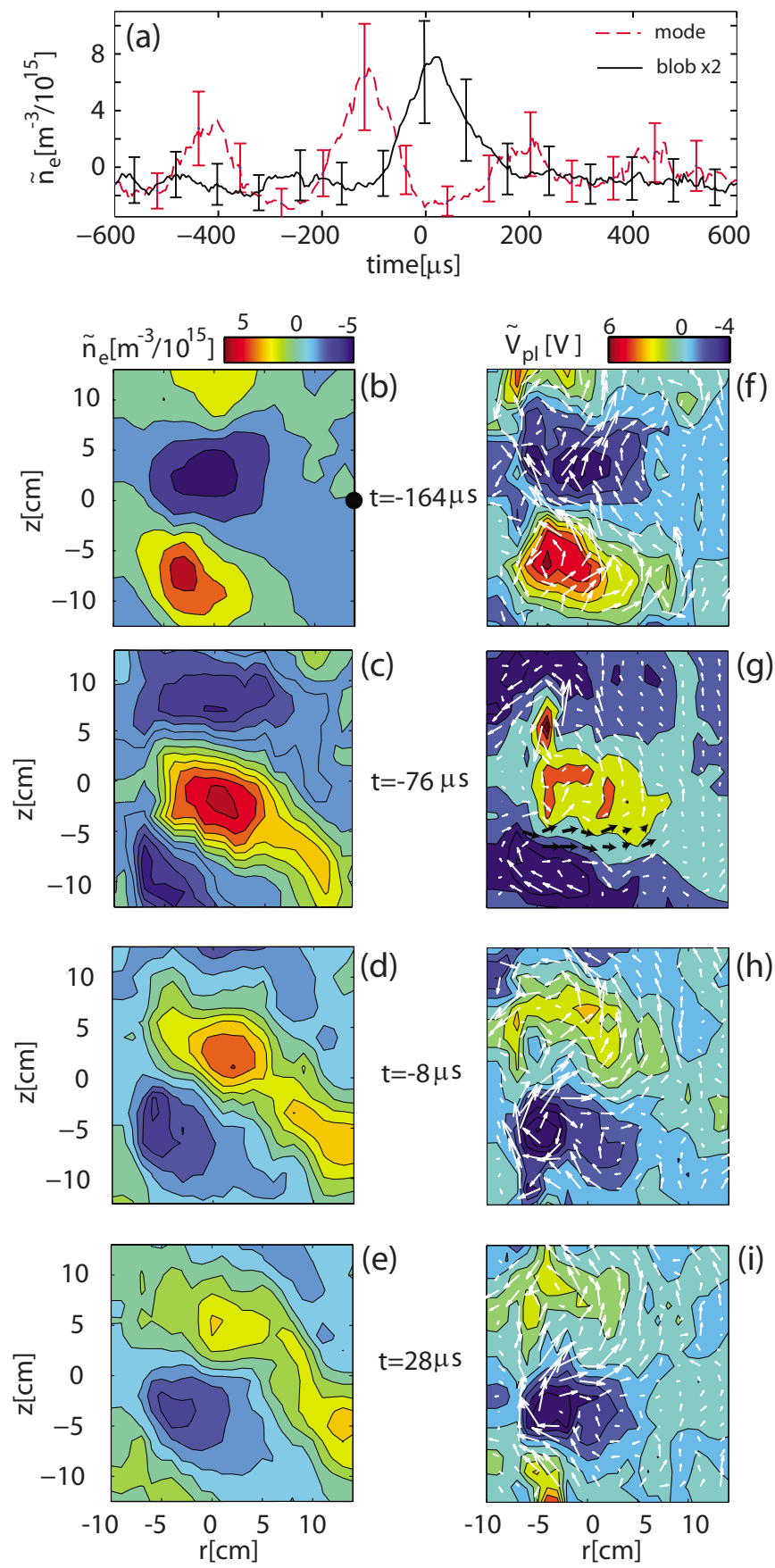

FIG. 5. (Color online) Plasma dynamics during blob ejection from the CS technique (a full animation is provided in Ref. 21). Shown are: (a) Time history of $\tilde{n}_{e}$, including uncertainties, in the mode region (dashed line) at $r=-1 \mathrm{~cm}, z=-5 \mathrm{~cm}$, and in the source free region (continuous line) at $r$ $=13 \mathrm{~cm}, z=-5 \mathrm{~cm}$. Note that this latter is multiplied by 2 for clarity. Twodimensional profiles of $\tilde{n}_{e}[(\mathrm{~b})-(\mathrm{e})]$ and of $\tilde{V}_{\mathrm{pl}}[(\mathrm{f})-(\mathrm{i})]$ at different times. Arrows show the instantaneous pattern of the total $\mathbf{v}_{E \times B}$. The black dot in frame (b) indicates the position $(r=14 \mathrm{~cm}, z=0)$ of the reference probe used for the CS technique, which is toroidally displaced by $90^{\circ}$ from the measuring plane shown here. In frame $(\mathrm{g})$, the location of the corridor, i.e., the zone where $\mathbf{v}_{E \times B}$ is directed mainly radially outward, is indicated by highlighted black arrows.

nonlinearities that saturates the interchange mode, which are not described by the linearized model.

In Fig. 5, frames (c)-(e) illustrate the dynamics of blob formation and ejection from the interchange wave. A radially elongated density structure, Fig. 5(c), forms from the posi- tive cell of the wave following from plasma convection by the $\mathbf{E} \times \mathbf{B}$ flow in a corridor, i.e., a radially extended region in which $\mathbf{v}_{E \times B}$ is mainly in the radial direction, as shown in Fig. $5(\mathrm{~g})$ by highlighted black arrows. This corridor extends radially over several ion sound radii $\left(\rho_{s} \approx 0.3 \mathrm{~cm}\right)$. In Figs. 5(d) and 5(e), the high- and low-field side parts of the elongated density structure are convected by the sheared $\mathbf{E} \times \mathbf{B}$ flow, respectively, at $\approx 1.2 \mathrm{~km} / \mathrm{s}$ and $\approx 0.4 \mathrm{~km} / \mathrm{s}$, such that a relative displacement between them is obtained. Eventually, the original density structure breaks into two parts, Fig. 5(e), thus forming a plasma blob on the low field side. We note that the structure in Fig. 5(d) is detected on the reference probe at $t \approx 0$, consistently with the blob being a filamentlike structure with $k_{\|} \approx 0$ elongated along the magnetic field line.

To quantify the role of the $\mathbf{E} \times \mathbf{B}$ flow in shearing off the density structure, we compute a shearing time ${ }^{29} 1 / \tau_{\text {sh }}$ $=\left(k_{z} L_{r} / 2 \pi\right) \partial v_{z, E \times B} / \partial r$, where $L_{r}$ and $v_{z, E \times B}$ are evaluated in the reference frame of the positive structure and then averaged over a transit time $\tau_{\text {tr }}=2 \pi /\left(k_{z} v_{z, E \times B}\right) \approx 200 \mu$ s during which the positive structure moves upwards by $2 \pi / k_{z}$. Here, $k_{z} \approx 30.6 \mathrm{~m}^{-1}$ is the wave number in the $z$ direction. We find a radial width of the shear layer $L_{r} \approx 0.03 \mathrm{~m}$ and $\left(\partial v_{z, E \times B}\right) / \partial r \approx 3 \times 10^{4} \mathrm{~s}^{-1}$, which result in a shearing time $\tau_{\mathrm{sh}} \approx 200 \mu \mathrm{s}$. This is of the same order of the transit time $\tau_{\mathrm{tr}}$, confirming that the radially elongated density structure, which forms from the wave, can be sheared off by the $\mathbf{E}$ $\times \mathbf{B}$ flow. The observed behavior is redolent of numerical simulations of edge/SOL turbulence in tokamaks, which show the generation of plasma blobs via shearing of density streamers by the plasma flow. ${ }^{12}$

To illustrate the mechanism driving the elongation of the density wave crest, we show in Fig. 6(a) a zoomed view of the instantaneous pattern of the fluctuating $\widetilde{\mathbf{v}}_{E \times B}$. In the same figure, solid gray lines display contours of the total electron pressure. Convective cells in the $\widetilde{\mathbf{v}}_{E \times B}$ field exchange zones of high plasma pressure with zones of low plasma pressure. This interchange mechanism has maximum drive where the local radial pressure gradient is maximum, which in the present case is in the region where the pressure negative wave crest is localized, Fig. 6(a). In Fig. 6(c), we show the time history of the $\left|L_{p e}^{-1}\right|$ maximum value across the measured spatial domain, as it evolves in the wave reference frame. At each time, we compute the 2D spatial Fourier transform $\tilde{n}_{e}\left(k_{r}, k_{z}\right)$ of the fluctuating density, Fig. 6(b). We compute a mode amplitude

$$
M=\sqrt{\sum_{k_{r}}\left|\tilde{n}_{e}\left(k_{r}, k_{z 0}\right)\right|^{2}},
$$

where $k_{z 0} \approx 30 \mathrm{~m}^{-1}$ and the summation is performed over all the computed radial wave numbers $k_{r}$. The time evolution of the mode amplitude is shown in Fig. 6(d). During a first period, $-600 \mu \mathrm{s}<t<-350 \mu \mathrm{s}$, both the mode amplitude and $\left|L_{p e}^{-1}\right|$ remain approximately constant. Later in time, $\left|L_{p e}^{-1}\right|$ is observed to increase from $\left|L_{p e}^{-1}\right| \approx 40 \mathrm{~m}^{-1}$ at $t \approx-350 \mu$ s to a maximum value $\left|L_{p e}^{-1}\right|_{\max } \approx 110 \mathrm{~m}^{-1}$ at $t \approx-260 \mu \mathrm{s}$. An increase of the mode amplitude follows which leads to a larger radial $\mathbf{v}_{E \times B}$ and to the elongation of the positive wave crest.

We investigate the link between the blob amplitude and the pressure scale length by performing the CS analysis over 

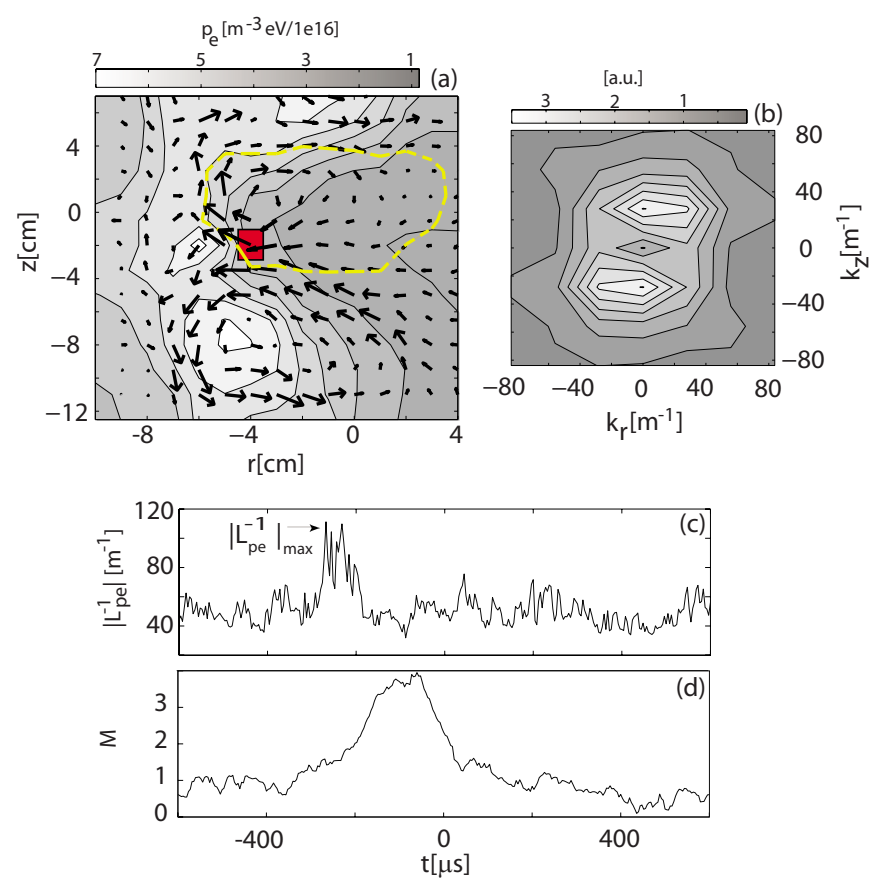

FIG. 6. (Color online) (a) A zoomed view of the instantaneous fluctuating $\mathbf{E} \times \mathbf{B}$ velocity field (arrows) at $t=-196 \mu$ s shows the convective cells interchanging zones of high and low plasma pressure. The square indicates the spatial location where $\left|L_{p e}^{-1}\right|$ is maximum at $t=-196 \mu \mathrm{s}$. The dashed line represents the contour where $\widetilde{p}_{e}=-0.1 \times 10^{16} \mathrm{~m}^{-3} \mathrm{eV}$ and identifies the position of the pressure negative wave crest. (b) Two-dimensional spatial Fourier transform $\widetilde{n}_{e}\left(k_{r}, k_{z}\right)$ at $t=-196 \mu \mathrm{s}$. (c) Time history of the $\left|L_{p e}^{-1}\right|$ maximum value across the measured spatial domain, as it evolves in the wave reference frame. (d) Time evolution of the mode amplitude, as defined in Eq. (4).

eight different classes of amplitudes defined by the conditions $(1+j / 2) \sigma \leqslant \tilde{I}_{\text {ref }} \leqslant(1.2+j / 2) \sigma$ with $j=0,1,2, \ldots, 7$. We find that the temporal evolution of $\left|L_{p e}^{-1}\right|$ and mode amplitude is similar to Fig. 6 for each class. Figure 7 shows a monotonic and faster than linear dependence of $\left|L_{p e}^{-1}\right|_{\max }$ upon the blob amplitude. The ejection of blobs is preceded by an increase of the pressure gradient. Blobs with larger amplitude are associated with larger $\left|L_{p e}^{-1}\right|_{\max }$. We define stationary gradients before and after the blob ejection by computing the averaged values of $\left|L_{p e}^{-1}\right|$ in a $200 \mu$ s time window centered, respectively, $400 \mu$ s before and after the blob event. These values do not show a strong dependence upon the blob amplitude, suggesting that the blob removes the excess of gradient and brings the profile back to the starting condition.

\section{SUMMARY AND OUTLOOK}

We have detailed experimentally a mechanism for blob generation from interchange waves in the toroidal magnetized plasma of the TORPEX device. The results show that a blob is generated from the wave crest of an interchange mode that increases in amplitude and expands radially following an increase of the local pressure gradient. The expansion of the mode forms a radially elongated structure that is eventually sheared off by the $\mathbf{E} \times \mathbf{B}$ flow, forming the blob.

Similar to the tokamak SOL, the magnetic configuration described here features open field lines, $\nabla B$, and magnetic field curvature. Blobs in TORPEX exhibit universal statisti-

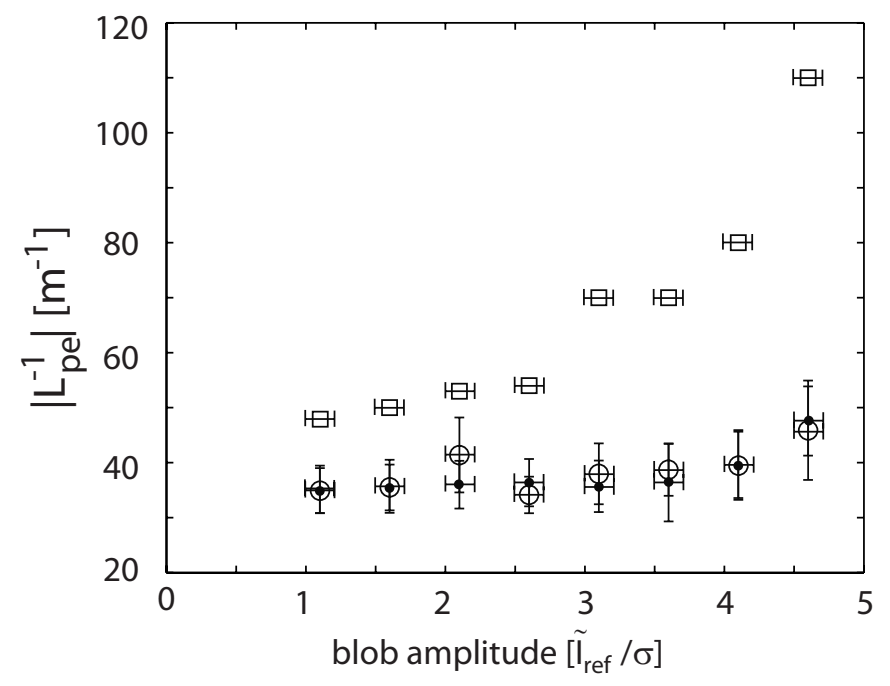

FIG. 7. (Color online) Dependence of various inverse pressure gradient scale lengths upon the blob size for the eight blob classes. Horizontal error bars represent the width of each blob class. Squares represent $\left|L_{p e}^{-1}\right|_{\max }$. Circles and dots are, respectively, the time-averaged values of $\left|L_{p e}^{-1}\right|$ before and after the blob event.

cal properties with strong similarities with observations in the tokamak SOL. ${ }^{27}$ Thus, the observed dynamics may shed light on the blob ejection mechanism in tokamaks, where there are strong indications that blobs result from interchange instabilities in the SOL. ${ }^{18}$ In tokamak geometry, a link between a local increase of the radial pressure gradient and the blob formation has recently been predicted for the edge region. ${ }^{30}$

Several experiments may now be envisaged in the TORPEX device to further investigate the physics of blobs. (1) We observe a transition from spectra dominated by coherent interchange modes at relatively high values of the vertical magnetic fields (such as in the present case) to spectra which are dominated by drift modes at lower values of vertical magnetic fields. ${ }^{31}$ The latter scenario provides a unique opportunity to investigate the role played by drift waves in the generation of blobs. (2) The identification of the blob generation mechanism may open new possibilities for controlling edge plasma transport. We are presently exploring the possibility of influencing the blob generation by biasing internal plates which may in turn modify the $\mathbf{E} \times \mathbf{B}$ velocity profile. (3) On the diagnostic side, a fast framing camera has been recently acquired for nonperturbing visible light measurements on TORPEX, which can be directly compared with local electrostatic measurements. (4) The present results detail a fundamental phenomenon in plasmas and represent an important test bed for validating both simulations and theoretical models of edge/SOL transport in toroidal plasmas. We have undertaken the development of a three-field fluid model in the TORPEX geometry which takes into account the experimentally determined heat and density sources. Code simulations will be benchmarked against experimental results for different source intensities and vertical magnetic fields, which can be directly controlled on TORPEX. 

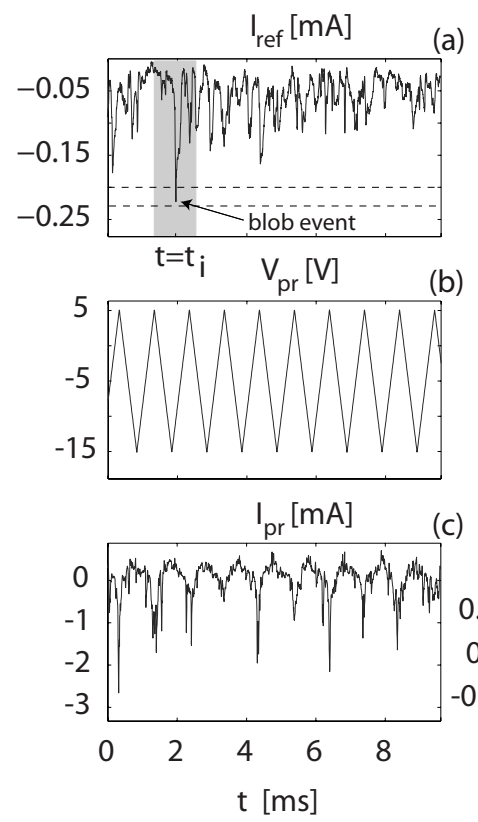

(c)
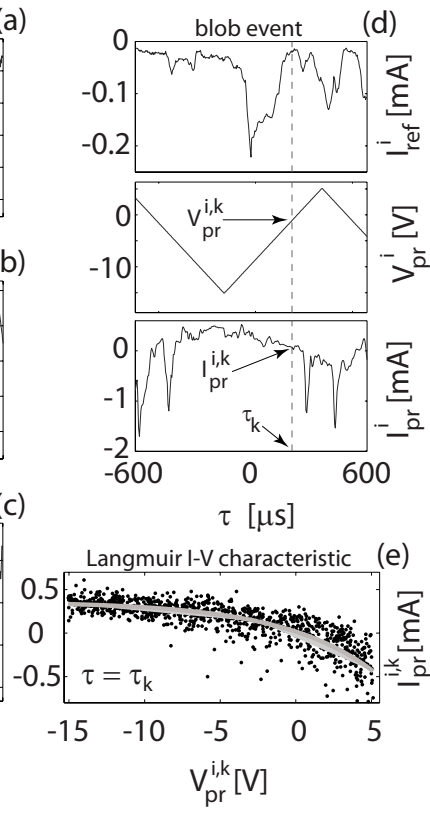

FIG. 8. (Color online) Illustration of the conditional sampling method. (a) Time series of ion saturation signal $I_{\text {ref }}$ from the reference probe at the edge. A blob of amplitude within the selected band (indicated by the two horizontal dashed lines) is detected at $t=t_{i}$. (b), (c) Sweeping voltage $V_{\mathrm{pr}}$ and measured current $I_{\mathrm{pr}}$ of a tip of the Langmuir probe array. The grey square in (a) indicates a reduced time window $-\Delta<\tau=t-t_{i}<\Delta$, with $\Delta=600 \mu \mathrm{s}$, in which time series (d) $I_{\mathrm{ref}}^{i}(\tau), V_{\mathrm{pr}}^{i}(\tau)$, and $I_{\mathrm{pr}}^{i}(\tau)$ are extracted. (e) The conditionally sampled $I-V$ Langmuir characteristics at $\tau=\tau_{k}$ (shown in grey) is reconstructed from the $\left[V_{\mathrm{pr}}^{i, k}, I_{\mathrm{pr}}^{i, k}\right]$ characteristics points for all $i$, i.e., all detected events.

\section{ACKNOWLEDGMENTS}

We thank F. Skiff, S. I. Krasheninnikov, and D. A. D'Ippolito for useful discussions.

This work is partly supported by the Fonds National Suisse de la Recherche Scientifique. P.R. is supported by a EURATOM Fusion fellowship.

\section{APPENDIX: CONDITIONAL SAMPLING METHOD}

Time-resolved 2D profiles of $n_{e}, T_{e}, V_{\mathrm{pl}}$ and velocity fields are needed to study the mechanism for blob generation, which cannot be obtained from HEXTIP measurements. In this paper, they are obtained by using a conditional sampling method allowing to reconstruct the $I-V$ Langmuir characteristic over many blob events. A general description of the standard CS technique can be found in Ref. 32. The method is based on two Langmuir probes. The first probe, an eight-tip, L-shaped Langmuir probe array, moves radially and rotates about its axis between discharges to cover a reduced $25 \times 20 \mathrm{~cm}^{2}$ section in the $r-z$ plane, Fig. 2(h). Each tip of the array is swept at $1 \mathrm{kHz}$ frequency in the range $V_{\mathrm{pr}}=[-15,+5] \mathrm{V}$ and the probe current $I_{\mathrm{pr}}$ is measured. The second Langmuir probe, dubbed reference probe, is left fixed in space at $r=14 \mathrm{~cm}, z=0$, and toroidally displaced by $90^{\circ}$ from the Langmuir probe array. The reference probe is biased at $V_{\text {ref }}=-40 \mathrm{~V}$ and measures an ion saturation signal $I_{\text {ref. }}$ All the signals are acquired at $250 \mathrm{kHz}$. In Fig. 8, we show the time traces of the reference signal $I_{\text {ref }}$ (a) and of $V_{\mathrm{pr}}$ and $I_{\mathrm{pr}}(\mathrm{b}, \mathrm{c})$ for one tip of the array. The reference signal is characterized by the presence of spikes due to the transit of a blob at the position of the probe. We use these spikes to select blobs. In the standard CS technique, events are selected requiring that the reference signal crosses a fixed threshold which is usually of a few standard deviations $\sigma$ from its mean value $\bar{I}_{\text {ref }}$. Here, we perform a selection of blobs over eight different classes of amplitudes defined by the conditions $(1+j / 2) \sigma \leqslant \widetilde{I}_{\text {ref }} \leqslant(1.2+j / 2) \sigma$ with $j=0,1,2, \ldots, 7$. This is used to investigate the link between the blob amplitude and the pressure scale length as discussed in Sec. IV. For each class, the $I_{\text {ref }}$ time series is searched for time intervals where the detection condition is satisfied and the times $t_{i}$, where $I_{\text {ref }}$ is maximum are defined as the blob detection times. In Fig. 8(a), a blob is detected at $t=t_{i}$ in the selected class, which is defined by two horizontal dashed lines. For the case in Fig. 5, approximately 600 blob events are found. For each event detected at $t=t_{i}$, we extract a reduced time window $-\Delta<\tau=t-t_{i}<\Delta$, with $\Delta=600 \mu \mathrm{s}$, in which time series (d) $I_{\mathrm{ref}}^{i}(\tau), V_{\mathrm{pr}}^{i}(\tau)$, and $I_{\mathrm{pr}}^{i}(\tau)$ are evaluated. For the blob detected in Fig. 8(a), an expanded view of the reduced time window is shown in Fig. 8(d). Since blobs are not synchronous with the sweeping signal, for each acquisition time $\tau=\tau_{k}$, the conditionally sampled $I-V$ Langmuir characteristics, shown in grey in Fig. 8(e), can be reconstructed from the $\left[V_{\mathrm{pr}}^{i, k}=V_{\mathrm{pr}}^{i}\left(\tau_{k}\right), I_{\mathrm{pr}}^{i, k}=I_{\mathrm{pr}}^{i}\left(\tau_{k}\right)\right]$ characteristics points for all $i$, i.e., all detected events. ${ }^{22,33}$ The conditionally sampled $I-V$ Langmuir characteristic is then used to extract $n_{e}, T_{e}$, and $V_{\mathrm{pl}}$. To increase the statistics, the $I-V$ characteristics is reconstructed using data from three identical $2.7 \mathrm{~s}$ long plasma discharges for each position of the L-shaped Langmuir probe array. By moving the L-shaped probe array between discharges, we obtain 2D profiles with $1 \mathrm{~cm}$ radial and $1.8 \mathrm{~cm}$ vertical resolution, from which the $\mathbf{E} \times \mathbf{B}$ velocity profile is reconstructed.

${ }^{1}$ J. A. Boedo, D. Rudakov, R. Moyer et al., Phys. Plasmas 8, 4826 (2001).

${ }^{2}$ S. J. Zweben, Phys. Fluids 28, 974 (1985).

${ }^{3}$ S. I. Krasheninnikov, Phys. Lett. A 283, 368 (2001).

${ }^{4}$ R. Sanchez, B. Ph. van Milligen, D. E. Newman, and B. A. Carreras, Phys. Rev. Lett. 90, 185005 (2003).

${ }^{5}$ M. Spolaore, V. Antoni, E. Spada, H. Bergsaker, R. Cavazzana, J. R. Drake, E. Marlines, G. Regnoli, G. Serianni, and N. Vianello, Phys. Rev. Lett. 93, 215003 (2004).

${ }^{6}$ G. Y. Antar, S. I. Krasheninnikov, P. Devynck, R. P. Doerner, E. M. Hollmann, J. A. Boedo, S. C. Luckhardt, and R. W. Conn, Phys. Rev. Lett. 87, 065001 (2001).

${ }^{7}$ T. A. Carter, Phys. Plasmas 13, 010701 (2006).

${ }^{8}$ T. Windisch, O. Grulke, and T. Klinger, Phys. Plasmas 13, 122303 (2006).

${ }^{9}$ M. Greenwald, Plasma Phys. Controlled Fusion 44, 27 (2002).

${ }^{10}$ T. D. Rognlien, R. H. Bulmer, M. E. Rensink, and J. N. Brooks, J. Nucl. Mater. 363-365, 658 (2007).

${ }^{11}$ S. J. Zweben, J. A. Boedo, O. Grulke, C. Hidalgo, B. LaBombard, R. J. Maqueda, P. Scarin, and J. L. Terry, Plasma Phys. Controlled Fusion 49, 1 (2007).

${ }^{12}$ N. Bisai, A. Das, S. Deshpande, R. Jha, P. Kaw, A. Sen, and R. Singh, Phys. Plasmas 11, 4018 (2004).

${ }^{13}$ W. Fundamenski, O. E. Garcia, V. Naulin, R. A. Pitts, A. H. Nielsen, J. J. Rasmussen, J. Horacek, and J. P. Graves, Nucl. Fusion 47, 417 (2007).

${ }^{14}$ D. A. Russell, D. A. D’Ippolito, J. R. Myra, W. M. Nevins, and X. Q. Xu, Phys. Rev. Lett. 93, 265001 (2004).

${ }^{15}$ O. E. Garcia, J. Horacek, R. A. Pitts, A. H. Nielsen, W. Fundamenski, J. P. Graves, V. Naulin, and J. Juul Rasmussen, Plasma Phys. Controlled Fusion 48, L1 (2006). 
${ }^{16}$ J. L. Terry, S. J. Zweben, K. Hallatschek et al., Phys. Plasmas 10, 1739 (2003).

${ }^{17}$ S. J. Zweben, R. J. Maqueda, D. P. Stotler et al., Nucl. Fusion 44, 134 (2004).

${ }^{18}$ J. R. Myra, D. A. D'Ippolito, D. P. Stotler, S. J. Zweben, B. P. LeBlanc, J. E. Menard, R. J. Maqueda, and J. Boedo, Phys. Plasmas 13, 092509 (2006).

${ }^{19}$ A. Fasoli, B. Labit, M. McGrath, S. H. Müller, G. Plyushchev, M. Podestà, and F. M. Poli, Bull. Am. Phys. Soc. 13, 119 (2003).

${ }^{20}$ S. H. Müller, A. Diallo, A. Fasoli, I. Furno, B. Labit, and M. Podestà, Phys. Plasmas 14, 110704 (2007).

${ }^{21}$ I. Furno, B. Labit, M. Podestà et al., Phys. Rev. Lett. 100, 055004 (2008).

${ }^{22}$ M. Podestà, A. Fasoli, B. Labit, M. McGrath, S. H. Müller, and F. M. Poli, Plasma Phys. Controlled Fusion 47, 1989 (2005).

${ }^{23}$ S. H. Müller, A. Fasoli, B. Labit, M. McGrath, O. Pisaturo, G. Plyushchev, M. Podestà, and F. M. Poli, Phys. Plasmas 12, 090906 (2005).

${ }^{24}$ J. M. Beall, Y. C. Kim, and E. J. Powers, J. Appl. Phys. 53, 3933 (1982).
${ }^{25}$ F. M. Poli, S. Brunner, A. Diallo, A. Fasoli, I. Furno, B. Labit, S. H. Müller, G. Plyushchev, and M. Podestà, Phys. Plasmas 13, 102104 (2006).

${ }^{26}$ A. Zeiler, J. F. Drake, and B. Rogers, Phys. Plasmas 4, 2134 (1997).

${ }^{27}$ B. Labit, I. Furno, A. Fasoli, A. Diallo, S. H. Müller, G. Plyushchev, M. Podestà, and F. M. Poli, Phys. Rev. Lett. 98, 255002 (2007).

${ }^{28}$ S. H. Müller, A. Diallo, A. Fasoli, I. Furno, B. Labit, G. Plyushchev, M. Podestà, and F. M. Poli, Phys. Plasmas 13, 100701 (2006).

${ }^{29}$ H. Biglari, P. H. Diamond, and P. W. Terry, Phys. Fluids B 2, 1 (1990).

${ }^{30}$ S. I. Krasheninnikov and A. I. Smolyakov, Phys. Plasmas 14, 102503 (2007).

${ }^{31}$ F. M. Poli, "Electrostatic instabilities and turbulence in a toroidal magnetized plasma," Ph.D. thesis, No. 3849, École Polytechnique Fédérale de Lausanne (2007). Available at http://library.epfl.ch/theses/?nr=3849.

${ }^{32}$ H. L. Pecseli and J. Trulsen, Phys. Fluids B 1, 1616 (1989).

${ }^{33}$ Th. Pierre, A. Escarguel, D. Guyomarc'h, R. Barni, and C. Riccardi, Phys. Rev. Lett. 92, 065004 (2004). 